\title{
Viruses and circadian rhythms
}

Many physiological processes are influenced or coordinated by 24-hour circadian rhythms, including processes within individual cells. Meanwhile, from Zika to the common cold, viruses are potentially dangerous pathogens that human bodies routinely encounter. These two areas of research, virology and circadian rhythms, might seem like disparate fields of biology; however, recent work performed by a University of Cambridge group (PNAS $113,10085-10090 ; 2016$ ) has demonstrated a novel interaction between viral infection and the circadian clock.

Interested in the relationship between viral pathogens and host circadian rhythm, Edgar and colleagues infected wild-type mice with a strain of herpes virus at different phases of the animals' circadian clocks. They found that viral replication was 10 times higher in the mice infected at the start of their resting phase, a phase analogous to late evenings for humans. The researchers then infected mice that were clock-deficient, $\mathrm{Bmal1}^{-/-}$mice, which lack evident circadian rhythms. These mice showed similar levels

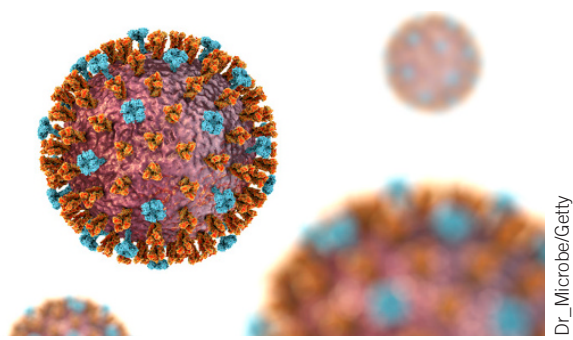

of viral replication when infected at different times of day, and within a week these circadian-deficient $\mathrm{Bmal1}^{-/-}$mice displayed viral replication that was over 3 times higher than wild type mice. These results show a clear relationship between circadian phase and virus infection, suggesting that timeof-day of infection plays a role in how well a virus will replicate inside its host.

The researchers considered the possibility that circadian rhythms could have affected the immune responses in the animals. Thus, they examined virus replication in cell models, where a systemic immune response was absent, and uncovered a surprising finding: even without a bodily immune system, the time of infection had a large influence on virus replication, just like it did in the living mice.

To examine whether the virus was capable of affecting the molecular rhythms of host cells, the researchers infected cells that express a bioluminescent marker controlled by the clock gene Bmal1 promoter, allowing the experimenters to directly observe how this clock gene is influenced by virus infection. Regardless of time-of-infection, Bmal1 expression was induced for about 6 hours after infection, and ensuing cellular circadian rhythms depended on the time at which the infection occurred. This finding suggests that viral infection can directly affect circadian clockwork within individual cells.

The result that viruses can interact with, and take advantage of, physiological rhythms provides new avenues of research into combating viruses, and also highlights a linkage between these seemingly separate research areas of biology.

James E. Niemeyer 